\title{
M-theory on Special Holonomy Spaces '
}

\author{
Mirjam Cvetič ${ }^{2 *}$, G.W. Gibbons ${ }^{3 \dagger}$, H. Lü ${ }^{4 * *}$ and C.N. Pope ${ }^{5 \ddagger}$ \\ ${ }^{*}$ Department of Physics and Astronomy \\ University of Pennsylvania, Philadelphia, PA 19104, USA \\ ${ }^{\dagger}$ DAMTP, Centre for Mathematical Sciences, Cambridge University \\ Wilberforce Road, Cambridge CB3 OWA, UK \\ ${ }^{* *}$ Michigan Center for Theoretical Physics, University of Michigan \\ Ann Arbor, MI 48109, USA \\ ${ }^{\ddagger}$ Center for Theoretical Physics, Texas A\&M University, College Station, TX 77843, USA
}

\begin{abstract}
We review the construction of regular $p$-brane solutions of M-theory and string theory with less than maximal supersymmetry whose transverse spaces have metrics with special holonomy, and where additional fluxes allow brane resolutions via transgression terms. We summarize the properties of resolved M2-branes and fractional D2-branes, whose transverse spaces are Ricci flat eight-dimensional and seven-dimensional spaces of special holonomy.
\end{abstract}

\section{INTRODUCTION}

Regular supergravity solutions with less than maximal supersymmetry may provide viable gravity duals to strongly coupled field theories with less than maximal supersymmetry. In particular, the regularity of such solutions at small distances sheds light on confinement and chiral symmetry breaking in the infrared regime of the dual strongly coupled field theory [1].

In this contribution we shall review the construction of such regular supergravity solutions with emphasis on resolved M2-branes of 11-dimensional supergravity and fractional D2-branes of Type IIA supergravity, which provide viable gravity duals of strongly coupled three-dimensional theories with $\mathrm{N}=2$ and $\mathrm{N}=1$ supersymmetry.

This construction has been referred to as a "resolution via transgression" [2]. It involves the replacement of the standard flat transverse space by a smooth space of special holonomy, i.e. a Ricci-flat space with fewer covariantly constant spinors. Furthermore,

\footnotetext{
1 Talk presented by M. Cvetič.

2 email:cvetic@cvetic.hep.upenn.edu

3 email:G.W.Gibbons@damtp.cam.ac.uk

4 email:hong!u@umich.edu

5 email:pope@physics.tamu.edu
}

CP607, String Theory, edited by H. Aoki and T. Tada (C) 2002 American Institute of Physics 0-7354-0051-2/02/\$19.00 
additional field strength contributions are involved, which are provided by harmonic forms in the space of special holonomy. Transgression-Chern-Simons terms modify the equation of motion and/or Bianchi identity for the original $p$-brane field strength. The construction will be reviewed for resolved M2-branes and D2-branes.

The explicit construction of such solutions has led to mathematical developments, for example obtaining harmonic forms for a large class of special holonomy metrics. As a prototype example we shall review the construction of the metric and the middledimensional forms for the Stenzel manifolds in $D=2 n$ (with $n \geq 2$ integer) [3,4]. We shall also briefly mention examples of known $G_{2}$ holonomy spaces and their associated harmonic forms. We also discuss the old as well as the new two-parameter metric with $\operatorname{Spin}(7)$ holonomy $[5,6]$ and the associated harmonic forms .

We then briefly summarize the properties of resolved M2-branes [2, 4] and fractional D2-branes $[2,7]$ as well as fractional M2-brane whose transverse space is that of the new $\operatorname{Spin}(7)$ holonomy metrics [5].

Finally, we summarize the key results and spell out directions for future work.

The work presented in this paper was initiated in [2] and further pursued in a series of papers that provide both new technical mathematical results and physics implications for resolved $p$-brane configurations $[3,4,5,6,7]$.

\section{RESOLUTION VIA TRANSGRESSION}

\section{Motivation}

The $A d S_{D+1} / C F T_{D}$ correspondence $[8,9,10]$ provides a quantitative insight into strongly coupled superconformal gauge theories in $D$ dimensions, by studying the dual supergravity solutions. The prototype supergravity dual is the D3-brane of Type IIB theory, with the classical solution

$$
\begin{aligned}
d s_{10}^{2} & =H^{-1 / 2} d x \cdot d x+H^{1 / 2}\left(d r^{2}+r^{2} d \Omega_{5}^{2}\right), \\
F_{(5)} & =d^{4} x \wedge d H^{-1}+\hat{*}\left(d^{4} x \wedge d H^{-1}\right), \\
\square H & =0 \Rightarrow H=1+\frac{R^{4}}{r^{4}} .
\end{aligned}
$$

In the decoupling limit $H=1+\frac{R^{4}}{r^{4}} \rightarrow \frac{R^{4}}{r^{4}}$ this reduces to $A d S_{5} \times S^{5}$, which provides a gravitational dual of the strongly coupled $\mathrm{N}=4$ super-Yang-Mills (SYM) theory.

Of course, the ultimate goal of this program is to elucidate strongly coupled YM theory, such as QCD, that has no supersymmetry. But for the time being important steps have been taken to obtain viable (regular) gravitational duals of strongly coupled field theories with less than maximal supersymmetry. In particular, within this framework we shall shed light on gravity duals of field theories in $D=\{2,3,4\}$ with $N=\{1,2\}$ supersymmetry.

As a side comment, within $D=5 \mathrm{~N}=2$ gauged supergravity progress has been made (see $[11,12]$ and references therein) in the explicit construction of domain wall solutions, both with vector-multiplets and hyper-multiplets, which lead to smooth solutions 
that provide viable gravity duals of $D=4 \mathrm{~N}=1$ conformal field theories. However, the aim in this contribution is to discuss the higher dimensional embeddings and the interpretation of such gravity duals.

A typical way to obtain a supergravity solution with lesser supersymmetry is to replace the flat transverse 6-dimensional space $d s_{6}^{2}=d r^{2}+r^{2} d \Omega_{5}^{2}$ of the D3-brane in (1) with a smooth non-compact Ricci-flat space with fewer Killing spinors. In this case the metric function $H$ still satisfies $\square H=0$, but now $\square$ is the Laplacian in the new Ricci-flat transverse space. This procedure ensures one has a solution with reduced supersymmetry; however the solution for $H$ is singular at the inner boundary of the transverse space, signifying the appearance of the (distributed) D3-brane source there.

A resolution of the singularity (and the removal of the additional source) can take place if one turns on additional fluxes ("fractional" branes). Within the D3-brane context, the Chern-Simons term of type IIB supergravity modifies the equations of motion:

$$
\begin{aligned}
d F_{(5)} & =d * F_{(5)}=F_{(3)}^{\mathrm{NS}} \wedge F_{(3)}^{\mathrm{RR}}=\frac{1}{2 i} F_{(3)} \wedge \bar{F}_{(3)}, \\
F_{(3)} & \equiv F_{(3)}^{\mathrm{RR}}+\mathrm{i} F_{(3)}^{\mathrm{NS}}=m L_{(3)},
\end{aligned}
$$

where $L_{(3)}$ is a complex harmonic self-dual 3-form on the 6-dimensional Ricci-flat space. Depending on the properties of $L_{3}$, this mechanism may allow for a smooth and thus viable supergravity solution. This is precisely the mechanism employed by Klebanov and Strassler, which in the case of the deformed conifold yields a supergravity dual of $D=4 \mathrm{~N}=1 \mathrm{SYM}$ theory. (For related and follow up work see, for example, $[13,14,15,16,17,18,19,20,21]$. For earlier work see, for example, [22, 23, 24, 25].)

In a general context the resolution via transgression [2] is a consequence of the ChernSimons-type (transgression) terms that are ubiquitous in supergravity theories. Such terms modify the Bianchi identities and/or equations of motion when additional field strengths are turned on. $p$-brane configurations with $(n+1)$-transverse dimensions, i.e. with "magnetic" field strength $F_{(n)}$, can have additional field strengths $F_{(p, q)}$ which, via transgression terms, modify the equations for $F_{(n)}$ :

$$
d F_{(n)}=F_{(p)} \wedge F_{(q)} ; \quad(p+q=n+1) .
$$

If the $(n+1)$-dimensional transverse Ricci-flat space admits a harmonic $p$-form $L_{(p)}$ then the equations of motion are satisfied if one sets $F_{(p)}=m L_{(p)}$, and by duality $F_{(q)} \sim \mu * L_{(p)}$. Depending on the $L^{2}$ normalizability properties of $L_{(p)}$, one may be able to obtain resolved (non-singular) solutions.

\section{Resolved M2-brane}

The transgression term in the 4-form field equation in 11-dimensional supergravity is given by

$$
d * F_{(4)}=\frac{1}{2} F_{(4)} \wedge F_{(4)},
$$

and the modified M2-brane Ansatz takes the form

$$
d \hat{s}_{11}^{2}=H^{-2 / 3} d x^{\mu} d x^{v} \eta_{\mu v}+H^{1 / 3} d s_{8}^{2},
$$




$$
F_{(4)}=d^{3} x \wedge d H^{-1}+m L_{(4)},
$$

where $L_{(4)}$ is a harmonic self-dual 4-form in the 8-dimensional Ricci-flat transverse space. The equation for $H$ is ten given by

$$
\square H=-\frac{1}{48} m^{2} L_{(4)}^{2} .
$$

For related work see, for example, [17, 27, 28, 29, 30, 31].

\section{Resolved D2-brane}

The transgression modification in the 4-form field equation in type IIA supergravity is

$$
d\left(e^{\frac{1}{2} \phi} \hat{*} F_{4}\right)=F_{(4)} \wedge F_{(3)},
$$

and the modified D2-brane Ansatz takes the form:

$$
\begin{aligned}
d s_{10}^{2} & =H^{-5 / 8} d x^{\mu} d x^{v} \eta_{\mu v}+H^{3 / 8} d s_{7}^{2}, \\
F_{(4)} & =d^{3} x \wedge d H^{-1}+m L_{(4)}, \quad F_{(3)}=m L_{(3)}, \quad \phi=\frac{1}{4} \log H,
\end{aligned}
$$

where $G_{(3)}$ is a harmonic 3-form in the Ricci-flat 7-metric $d s_{7}^{2}$, and $L_{(4)}=* L_{(3)}$, with $*$ the Hodge dual with respect to the metric $d s_{7}^{2}$. The function $H$ satisfies

$$
\square H=-\frac{1}{6} m^{2} L_{(3)}^{2},
$$

where $\square$ denotes the scalar Laplacian with respect to the transverse 7-metric $d s_{7}^{2}$. Thus the deformed D2-brane solution is completely determined by the choice of Ricci-flat 7-manifold, and the harmonic 3-form supported by it.

\section{Other Examples}

In general the transgression terms modify field equations or Bianchi identities as given in (3), thus allowing resolved branes with $(n+1)$ transverse dimensions for the following additional examples in M-theory and string theory:

- (i) D0-brane: $d * F_{(2)}=* F_{(4)} \wedge F_{(3)}$,

- (ii) D1-brane: $d * F_{(3)}^{\mathrm{RR}}=F_{(5)} \wedge F_{(3)}^{\mathrm{NS}}$,

- (iii) D4-brane: $d F_{(4)}=F_{(3)} \wedge F_{(2)}$,

- (iv) IIA string: $d * F_{(3)}=F_{(4)} \wedge F_{(4)}$,

- (v) IIB string: $d * F_{(3)}^{\mathrm{NS}}=F_{(5)} \wedge F_{(3)}^{\mathrm{RR}}$,

- (vi) heterotic 5-brane: $d F_{(3)}=F_{(2)}^{i} \wedge F_{(2)}^{i}$.

In what follows, we shall focus on resolved M2-branes and briefly mention fractional D2-branes. For details of other examples and their properties, see e.g., [2, 3, 32]. 


\section{MATHEMATICAL DEVELOPMENTS}

The construction of resolved supergravity solutions necessarily involves the explicit form of the metric on the Ricci-flat special holonomy spaces. These spaces fall into the following classes:

- Kähler spaces in $D=2 n$ dimensions ( $n$-integer) with $S U(n)$ holonomy, and two covariantly constant spinors. There are many examples, with the Stenzel metric on $T^{*} S^{n}$ providing a prototype. They are typically asymptotically conical (AC).

- Hyper-Kähler spaces in $D=4 n$ with $S p(n)$ holonomy, and $n+1$ covariantly constant spinors. Subject to certain technical assumptions, Calabi's metric on the cotangent bundle of $\mathbf{C P}^{n}$ is the only complete irreducible cohomogeneity one example [33].

- In $D=7$ there are exceptional $G_{2}$ holonomy spaces with one covariantly constant spinor. Until recently only three AC examples were known [34, 35], but new metrics have been recently constructed in $[37,36,38,39,40]$.

- In $D=8$ there are exceptional $\operatorname{Spin}(7)$ holonomy spaces with one covariantly constant spinor; until recently only one AC example was known [34, 35]. New metrics were recently constructed in $[5,6]$.

The focus is on a construction of cohomogeneity one spaces that are typically asymptotic to cones over Einstein spaces. Recent mathematical developments evolved along two directions: (i) construction of harmonic forms on known Ricci-flat spaces (see in particular [3, 7]), (ii) construction of new exceptional holonomy spaces $[5,6,36,37,38$, $39,40]$. In the following two subsections we illustrate these developments by summarizing (i) results on the construction of harmonic forms on the Stenzel metric [3] and briefly mention $G_{2}$ holonomy metrics [2,7], and (ii) results for the construction of new $\operatorname{Spin}(7)$ two-parameter metrics [5, 6], all serving as prototype examples.

\section{Harmonic forms for the Stenzel metric}

The Stenzel[41] construction provides a class of complete non-compact Ricci-flat Kähler manifolds, one for each even dimension, on the co-tangent bundle of the $(n+1)$ sphere, $T^{\star} S^{n+1}$. These are asymptotically conical, with principal orbits that are described by the coset space $S O(n+2) / S O(n)$, and they have real dimension $d=2 n+2$.

\section{Stenzel metric}

In the following we summarize the relevant results for the construction of the Stenzel metric. (For more details see [3].) This construction [3, 41] of the Stenzel metric starts with $L_{A B}$, which are left-invariant 1-forms on the group manifold $S O(n+2)$. By splitting the index as $A=(1,2, i)$, we have that $L_{i j}$ are the left-invariant 1 -forms for the $S O(n)$ 
subgroup, and so the 1-forms in the coset $S O(n+2) / S O(n)$ will be

$$
\sigma_{i} \equiv L_{1 i}, \quad \tilde{\sigma}_{i} \equiv L_{2 i}, \quad v \equiv L_{12} .
$$

The metric Ansatz takes the form:

$$
d s^{2}=d t^{2}+a^{2} \sigma_{i}^{2}+b^{2} \tilde{\sigma}_{i}^{2}+c^{2} v^{2},
$$

where $a, b$ and $c$ are functions of the radial coordinate $t$. One defines Vielbeine

$$
e^{0}=d t, \quad e^{i}=a \sigma_{i}, \quad e^{\tilde{i}}=b \tilde{\sigma}_{i}, \quad e^{\tilde{0}}=c \nu,
$$

for which one can introduce a holomorphic tangent-space basis of complex 1-forms $\varepsilon^{\alpha}$ :

$$
\varepsilon^{0} \equiv-e^{0}+\mathrm{i} e^{\tilde{0}}, \quad \varepsilon^{i}=e^{i}+\mathrm{i} e^{\tilde{i}} .
$$

Defining $a=e^{\alpha}, b=e^{\beta}, c=e^{\gamma}$, and introducing the new coordinate $\eta$ by $a^{n} b^{n} c d \eta=d t$, one finds [3] that the Ricci-flat equations can be obtained from a Lagrangian $L=T-V$ which can be written as a "supersymmetric Lagrangian": $L=\frac{1}{2} g_{i j}\left(d \alpha^{i} / d \eta\right)\left(d \alpha^{j} / d \eta\right)-\frac{1}{2} g^{i j} \frac{\partial W}{\partial \alpha^{i}} \frac{\partial W}{\partial \alpha^{j}}$. The solution of the first-order equations yields the explicit solution:

$$
a^{2}=R^{1 /(n+1)} \operatorname{coth} r, b^{2}=R^{1 /(n+1)} \tanh r, h^{2}=c^{2}=\frac{1}{n+1} R^{-n /(n+1)}(\sinh 2 r)^{n},
$$

where $R(r) \equiv \int_{0}^{r}(\sinh 2 u)^{n} d u$, and the radial coordinate $r$ is introduced as $d t=h d r$.

For each $n$ the result is expressible in relatively simple terms. For example:

$$
n=1: R=\sinh ^{2} r ; n=2: R=\frac{1}{8}(\sinh 4 r-4 r) ; n=3: R=\frac{2}{3}(2+\cosh 2 r) \sinh ^{4} r .
$$

The case $n=1$ is the Eguchi-Hanson metric [26], and $n=2$ it is the deformed conifold [42].

As $r$ approaches zero, the metric takes the form

$$
d s^{2} \sim d r^{2}+r^{2} \tilde{\sigma}_{i}^{2}+\sigma_{i}^{2}+v^{2},
$$

which has the structure locally of the product $\mathbf{R}^{n+1} \times S^{n+1}$, with $S^{n+1}$ being a "bolt." As $r$ tends to infinity, the metric becomes

$$
d s^{2} \sim d \rho^{2}+\rho^{2}\left\{\frac{n^{2}}{(n+1)^{2}} v^{2}+\frac{n}{2(n+1)^{2}}\left(\sigma_{i}^{2}+\tilde{\sigma}_{i}^{2}\right)\right\},
$$

representing a cone over the Einstein space $S O(n+2) / S O(n)$.

\section{Harmonic middle-dimension $(p, q)$ forms}

An Ansatz compatible with the symmetries of the Stenzel metric is of the form:

$$
\begin{aligned}
L_{(p, q)}= & f_{1} \varepsilon_{i_{1} \cdots i_{q-1} j_{1} \cdots j_{p}} \bar{\varepsilon}^{0} \wedge \bar{\varepsilon}^{i_{1}} \wedge \cdots \wedge \bar{\varepsilon}^{i_{q-1}} \wedge \varepsilon^{j_{1}} \wedge \cdots \wedge \varepsilon^{j_{p}} \\
& +f_{2} \varepsilon_{i_{1} \cdots i_{p-1} j_{1} \cdots j_{q}} \varepsilon^{0} \wedge \varepsilon^{i_{1}} \wedge \cdots \wedge \varepsilon^{i_{p-1}} \wedge \bar{\varepsilon}^{j_{1}} \wedge \cdots \wedge \bar{\varepsilon}^{j_{q}},
\end{aligned}
$$


with $f_{1}, f_{2}$ being functions of $r$, only. The harmonicity condition becomes $d L_{(p, q)}=$ 0 , since $* L_{(p, q)}=\mathrm{i}^{p-q} L_{(p, q)}$. The functions $f_{1}, f_{2}$ are solutions of coupled first-order homogeneous differential equations, yielding a solution that is finite as $r \rightarrow 0$ :

$$
\begin{gathered}
f_{1}=q_{2} F_{1}\left[\frac{1}{2} p, \frac{1}{2}(q+1), \frac{1}{2}(p+q)+1 ;-(\sinh 2 r)^{2}\right], \\
f_{2}=-p_{2} F_{1}\left[\frac{1}{2} q, \frac{1}{2}(p+1), \frac{1}{2}(p+q)+1 ;-(\sinh 2 r)^{2}\right] .
\end{gathered}
$$

For any specific integers $(p, q)$, these are elementary functions of $r$.

For the two special cases of greatest interest, they have the following properties:

- $(p, p)$-forms in $4 p$-dimensions: $f_{1}=-f_{2}=\frac{p}{(\cosh r)^{2 p}}$ with $\left|L_{(p, p)}\right|^{2}=\frac{\text { const. }}{(\cosh r)^{4 p}}$ fallsoff fast enough as $r \rightarrow \infty$. This turns out to be the only $L^{2}$ normalizable form.

- $(p+1, p)$-forms in $(4 p+2)$-dimensions. As $r \rightarrow \infty:\left|L_{(p+1, p)}\right|^{2} \sim \frac{1}{[\sinh (2 r)]^{2 p}}$ which is marginally $L^{2}$-non-normalizable.

As for physics implications, the case in $2(n+1)=4$ dimensions with an $L^{2}$ normalizable $L_{(1,1)}$-form is precisely the example of the resolved self-dual string discussed in detail in Section 2.2.

In $2(n+1)=6$ dimensions, the $L_{(2,1)}$-form was constructed in [1], and provides a resolution of the D3-brane. Since $L_{(2,1)}$ is only marginaliy non-normalizable as $r \rightarrow \infty$, the decoupling limit of the space-time does not give an $\mathrm{AdS}_{5}$, but instead there is a logarithmic modification. In particular, this modification accounts for a renormalization group running of the difference of the inverse-squares of the two gauge group couplings in the dual $S U(N) \times S U(N+M)$ SYM [24].

On the other hand in $2(n+1)=8$ dimensions the $L^{2}$ normalizable $L_{(2,2)}$-form supports additional fluxes that resolve the original $M 2$-brane, whose details will be given in Section 5.1 .

It turns out that one can construct regular supersymmetric resolved M2-branes for many other examples of 8-dimensional special holonomy transverse spaces, such as the original $\operatorname{Spin}(7)$ holonomy transverse space [2], a number of new Kähler spaces [2, 7], and hyper-Kähler spaces [4].

\section{Old $G_{2}$ holonomy metrics and their harmonic forms}

$$
\text { Resolved cones over } S^{2} \times S^{4} \text { and } S^{2} \times \mathbf{C} \mathbf{P}^{2}
$$

The first type of complete Ricci-flat 7-dimensional metrics of $G_{2}$ holonomy, obtained in [34, 35], correspond to $R^{3}$ bundles over four-dimensional quaternionic-Kähler Einstein base manifolds $M$. These spaces are of cohomogeneity one, with level surfaces that are $S^{2}$ bundles over $M$ (also known as "twistor spaces" over $M$ ). There are two cases that arise, with $M$ being $S^{4}$ or $\mathbf{C P}^{2}$. Thus the two manifolds have level surfaces that are $\mathbf{C P}^{3}\left(S^{2}\right.$ bundle over $\left.S^{4}\right)$ or the flag manifold $S U(3) /(U(1) \times U(1))\left(S^{2}\right.$ bundle over $\mathbf{C P}^{2}$ ), respectively. These two manifolds are the bundles of self-dual 2-forms over $S^{4}$ or $\mathbf{C} \mathbf{P}^{2}$ respectively. They approach $\mathbf{R}^{3} \times S^{4}$ or $\mathbf{R}^{3} \times \mathbf{C P}^{2}$ locally near the origin. (The 
calculations for the two cases, with the principal orbits being $S^{2}$ bundles either over $S^{4}$ or over $\mathbf{C P}^{2}$, proceed essentially identically.)

The harmonic 3-form, was constructed in [4]: it is a fully $L^{2}$-normalizable.

\section{Resolved cone over $S^{3} \times S^{3}$}

The remaining complete 7-dimensional manifold of $G_{2}$ holonomy obtained in [34, 35] is again of cohomogeneity one, with principal orbits are topologically $S^{3} \times S^{3}$. The manifold is the spin bundle of $S^{3}$; near the origin it approaches locally $\mathbf{R}^{4} \times S^{3}$.

This metric admits a regular harmonic 3-form, explicitly constructed in [2]: it is square-integrable at short distance, but gives a linearly divergent integral at large distance. The short-distance square-integrability is enough to give a regular deformed D2brane solution, even though $L_{(3)}$ is not $L^{2}$-normalizable.

\section{New $\operatorname{Spin}(7)$ holonomy metrics}

\section{The old metric and harmonic 4-forms}

Until recently only one explicit example of a complete non-compact metric on a $\operatorname{Spin}(7)$ holonomy space was known $[34,35]$. The principal orbits are $S^{7}$, viewed as an $S^{3}$ bundle over $S^{4}$. The solution (21) is asymptotic to a cone over the "squashed" Einstein 7-sphere, and it approaches $\mathbf{R}^{4} \times S^{4}$ locally at short distance (i.e. $r \approx \ell$ ). The metric is of the form:

$$
d s_{8}^{2}=\left(1-\frac{\ell^{10 / 3}}{r^{10 / 3}}\right)^{-1} d r^{2}+\frac{9}{100} r^{2}\left(1-\frac{\ell^{10 / 3}}{r^{10 / 3}}\right) h_{i}^{2}+\frac{9}{20} r^{2} d \Omega_{4}^{2},
$$

where $h_{i} \equiv \sigma_{i}-A_{(1)}^{i}$, and the $\sigma_{i}$ are left-invariant 1-forms on $S U(2), d \Omega_{4}^{2}$ is the metric on the unit 4-sphere, and $A_{(1)}^{i}$ is the $S U(2)$ Yang-Mills instanton on $S^{4}$. The $\sigma_{i}$ can be written in terms of Euler angles as

$\sigma_{1}=\cos \psi d \theta+\sin \psi \sin \theta d \varphi, \quad \sigma_{2}=-\sin \psi d \theta+\cos \psi \sin \theta d \varphi, \quad \sigma_{3}=d \psi+\cos \theta d \varphi$.

A regular $L^{2}$ normalizable harmonic 4-form in this metric was obtained in [2].

\section{New Spin(7) holonomy metric}

The generalization that we shall consider involves allowing the $S^{3}$ fibers of the previous construction themselves to be "squashed." Namely, the $S^{3}$ bundle is itself written as a $U(1)$ bundle over $S^{2}$ leading to the following "twice squashed" Ansatz:

$$
d \hat{s}_{8}^{2}=d t^{2}+a^{2}\left(D \mu^{i}\right)^{2}+b^{2} \sigma^{2}+c^{2} d \Omega_{4}^{2},
$$


where $a, b$ and $c$ are functions of the radial variable $t$. (The previous $\operatorname{Spin}(7)$ example has $a=b$.) Here

$$
\mu_{1}=\sin \theta \sin \psi, \quad \mu_{2}=\sin \theta \cos \psi, \quad \mu_{3}=\cos \theta,
$$

are the $S^{2}$ coordinates, subject to the constraint $\mu_{i} \mu_{i}=1$, and

$$
D \mu^{i} \equiv d \mu^{i}+\varepsilon_{i j k} A_{(1)}^{j} \mu^{k}, \quad \sigma \equiv d \varphi+\mathrm{A}_{(1)}, \quad \mathrm{A}_{(1)} \equiv \cos \theta d \psi-\mu^{i} A_{(1)}^{i},
$$

where the field strength $\mathrm{F}_{(2)}$ of the $U(1)$ potential $\mathrm{A}_{(1)}$ turns out to be given by: $\mathrm{F}_{(2)}=\frac{1}{2} \varepsilon_{i j k} \mu^{k} D \mu^{i} \wedge D \mu^{j}-\mu^{i} F_{(2)}^{i}$.

The Ricci-flatness conditions can be satisfied by solving the first-order equations of a supersymmetric Lagrangian, yielding the following special solution (for details see $[5,6])$ :

$d s_{8}^{2}=\frac{(r-\ell)^{2} d r^{2}}{(r-3 \ell)(r+\ell)}+\frac{\ell^{2}(r-3 \ell)(r+\ell)}{(r-\ell)^{2}} \sigma^{2}+\frac{1}{4}(r-3 \ell)(r+\ell)\left(D \mu^{i}\right)^{2}+\frac{1}{2}\left(r^{2}-\ell^{2}\right) d \Omega_{4}^{2}$,

The quantity $\frac{1}{4}\left[\sigma^{2}+\left(D \mu^{i}\right)^{2}\right]$ is the metric on the unit 3-sphere, and so in this case we find that the metric smoothly approaches $\mathbf{R}^{4} \times S^{4}$ locally, at small distance $(r \rightarrow 3 \ell)$, i.e. it has the same topology as the old $\operatorname{Spin}(7)$ holonomy space. On the other hand, it locally approaches $\mathrm{M}_{7} \times S^{1}$ at large distance. Here $\mathrm{M}_{7}$ denotes the 7-manifold of $G_{2}$ holonomy that is the $\mathbf{R}^{3}$ bundle over $S^{4}[34,35]$. Asymptotically the new metric behaves like a circle bundle over an asymptotically conical manifold in which the length of the $U(1)$ fibers tends to a constant; in other words, it is ALC.

If one takes $r$ to be negative, or instead analytically continues the solution so that $\ell \rightarrow-\ell$ (keeping $r$ positive), one gets a different complete manifold. Thus instead of (26), the quantity $\frac{1}{4}\left(\sigma^{2}+\left(D \mu^{i}\right)^{2}+d \Omega_{4}^{2}\right)$ is precisely the metric on the unit 7-sphere, and so as $r$ approaches $\ell$ the metric $d s_{8}^{2}$ smoothly approaches $\mathbf{R}^{8}$. At large $r$ the function $b$, which is the radius in the $U(1)$ direction $\sigma$, approaches a constant, and so the metric tends to an $S^{1}$ bundle over a 7 -metric of the form of a cone over $\mathbf{C P}^{3}$; it has the same asymptotic form as (26). The manifold in this case is topologically $\mathbf{R}^{8}$.

In $[5,6]$ the general solution to the first-order system of equations is obtained, leading to additional families of regular metrics of $\operatorname{Spin}(7)$ holonomy, which are complete on manifolds $\mathbf{B}_{8}^{ \pm}$that are similar to $\mathbf{B}_{8}$. These additional metrics have a non-trivial integration constant which parameterizes inequivalent solutions. (For details see [6] and Appendix A of [5]).

$L^{2}$ normalizable harmonic 4-forms for the new $\operatorname{Spin}(7)$ 8-manifolds were obtained in [5].

\section{Applications: resolved M2- and D2-branes}

The explicit construction of harmonic four-forms of 8-dimensional Ricci flat spaces led to the analytic expressions of resolved M2-brane solutions, while the three-forms 
(and dual four-forms) of $G_{2}$ holonomy spaces to analytic expressions for fractional D2branes. Their properties such as supersymmetry conditions, flux integrals and aspects of dual field theories were discussed in particular in [2, 3, 4, 31] and [7], respectively.

Resolved M2-branes are always supported by $L^{2}$-normalizable harmonic forms and thus they are regular at short distance and have decoupling limits at large distance that yield $A d S_{4}$. They have no conserved additional (fractional) charges. The dual 3dimensional dual field theory is therefore a superconformal field theory (with $\mathrm{N}=1$ or $\mathrm{N}=2$ supersymmetry) which is in turn perturbed by marginal operators associated with pseudo-scalar fields [31]. On the other hand the fractional D2-branes have conserved fractional charges corresponding to D4-branes wrapping the 2-cycles dual to $S^{4}\left(\mathbf{C P}^{2}\right)$ in $\mathrm{M}_{7}$, or to NS-NS 5-branes wrapping the 3-cycle dual to $S^{3}$ in $\mathrm{M}_{7}$, respectively.

An interesting implication involves the properties of fractional M2-branes using the new $\operatorname{Spin}(7)$ holonomy spaces. After reduction on $S^{1}$ these give fractional D2-branes where, whose fractional magnetic charge is for D4-branes wrapping 2-cycles and for D6-branes wrapping 4-cycles. The fact that the resolved M2-brane on the new Spin(7) holonomy space has non-zero fractional charge is a consequence of the asymptotically locally conical structure of the new $\operatorname{Spin}(7)$ holonomy space.

\section{CONCLUSIONS AND OPEN AVENUES}

In this contribution we have presented a summary of some recent developments in the construction of regular $p$-brane configurations with less than maximal supersymmetry. In particular, the method involves the introduction of complete non-compact special holonomy metrics and additional fluxes, supported by harmonic-forms in special holonomy spaces, which modify the original $p$-brane solutions via Chern-Simons (transgression) terms.

The work led to a number of important mathematical developments which we have also summarized. Firstly, the construction of harmonic forms for special holonomy spaces in diverse dimensions was reviewed, and the explicit construction of harmonic forms for Stenzel metrics was summarized. Secondly, a construction of new twoparameter $\operatorname{Spin}(7)$ holonomy spaces was discussed. These have the property that they interpolate asymptotically between a local $S^{1} \times \mathrm{M}_{7}$, where the length of the circle is finite and $\mathrm{M}_{7}$ is the $G_{2}$ holonomy space with the topology of the $S^{2}$ bundle over $S^{4}$, while at small distance they approach the "old" $\operatorname{Spin}(7)$ holonomy space with the topology of the chiral spin bundle over $S^{4}$.

The mathematical developments also led to a number of important physics implications, relevant for the properties of the resolved $p$-brane solutions, briefly summarized in the section above. In particular, the focus was on the properties of resolved M2-branes with 8-dimensional special holonomy transverse spaces (e.g., Stenzel, hyper-Kähler and $\operatorname{Spin}(7)$ holonomy spaces) and the results for the fractional D2-branes with three 7dimensional $G_{2}$ holonomy transverse spaces.

There are a number of open avenues in the exploration of further properties of such solutions. In particular, it is of importance to study the properties of the dual three dimensional field theories in greater detail. 
Another important direction involves the study of novel special holonomy spaces in M-theory. In particular, novel constructions of $G_{2}$ holonomy spaces [36, 37, 38, 39, 40] are of importance in the study of $N=1 \mathrm{D}=4$ field theory aspects of M-theory [43, $44,45]$ and the study of M-theory on spaces of $G_{2}$ holonomy has recently attracted considerable attention. Specifically, it has been proposed that $\mathrm{M}$-theory compactified on a certain singular seven-dimensional space with $G_{2}$ holonomy might be related to an $\mathrm{N}=1, D=4$ gauge theory $[10,43,44,45,47]$ that has no conformal symmetry. The quantum aspects of M-theory dynamics on spaces of $G_{2}$ holonomy can provide insights into non-perturbative aspects of four-dimensional $\mathrm{N}=1$ field theories, such as the preservation of global symmetries and phase transitions. For example, Ref. [43] provides an elegant exposition and study of these phenomena for the three manifolds of $G_{2}$ holonomy that were obtained in $[34,35]$.

A recent development in this direction is the discovery of M3-brane configurations $[48,36]$ which have a flat 4-dimensional world-volume and the transverse space that is a deformation of the $G_{2}$ along with the 4-form field strength turned on. These configurations turn out to have zero charge and ADM mass (leading to naked singularities at small distances).

\section{ACKNOWLEDGEMENT}

Research is supported in part by DOE grant DE-FG02-95ER40893, NSF grant No. PHY99-07949, Class of 1965 Endowed Term Chair and NATO Collaborative research grant 976951 (M.C.), in full by DOE grant DE-FG02-95ER40899 (H.L.) and in part by DOE grant DE-FG03-95ER40917 (C.P.).

\section{REFERENCES}

1. I.R. Klebanov and M.J. Strassler, Supergravity and a confining gauge theory: duality cascades and

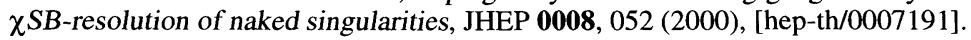

2. M. Cvetič, H. Lü and C.N. Pope, Brane resolution through transgression, Nucl. Phys. B 600, 103 (2001) [hep-th/0011023].

3. M. Cvetič, G.W. Gibbons, H. Lü and C.N. Pope, Ricci-flat metrics, harmonic forms and brane resolutions, hep-th/0012011.

4. M. Cvetič, G. W. Gibbons, H. Lü and C. N. Pope, Hyper-Kähler Calabi metric, $L^{2}$ harmonic forms, resolved M2-branes, and AdS $S_{4} / C F T_{3}$ correspondence, hep-th/0102185.

5. M. Cvetič, G. W. Gibbons, H. Lü and C. N. Pope, New complete non-compact Spin(7) manifolds, hep-th/0103155.

6. M. Cvetič, G.W. Gibbons, H. Lü and C.N. Pope, New cohomogeneity one metrics with Spin(7) holonomy, math.DG/0105119.

7. M. Cvetič, G. W. Gibbons, H. Lü and C. N. Pope, Supersymmetric non-singular fractional D2-branes and NS-NS 2-branes, hep-th/0101096, to appear in Nucl. Phys. B.

8. J. Maldacena, The large $N$ limit of superconformal field theories and supergravity, Adv. Theor. Math. Phys. 2 (1998) 231, hep-th/9711200.

9. S.S. Gubser, I.R. Klebanov and A.M. Polyakov, Gauge theory correlators from non-critical string theory, Phys. Lett. B428 (1998) 105, hep-th/9802109.

10. E. Witten, Anti-de Sitter space and holography, Adv. Theor. Math. Phys. 2 (1998) 253, hepth/980215. 
11. $\mathrm{K}$. Behrndt and $\mathrm{M}$. Cvetič, Gauging of $N=2$ supergravity hypermultiplet and novel renormalization group flows, hep-th/0101007.

12. A. Ceresole, G. Dall'Agata, R. Kallosh and A. Van Proeyen, Hypermultiplets, domain walls and supersymmetric attractors, hep-th/0104056.

13. M. Graña and J. Polchinski, Supersymmetric three-form flux perturbations on $A d S_{5}$, hep-th/0009211.

14. J. Maldacena and C. Nuñez, Supergravity description of field theories on curved manifolds and a no go theorem, Int. J. Mod. Phys. A 16, 822 (2001) [hep-th/0007018].

15. S.S. Gubser, Supersymmetry and F-theory realization of the deformed conifold with three-form flux, hep-th/0010010.

16. L.A. Pando Zayas and A.A. Tseytlin, 3-branes on a resolved conifold, hep-th/0010088.

17. K. Becker and M. Becker, Compactifying M-theory to four dimensions, JHEP 0011, 029 (2000) [hep-th/0010282].

18. M. Bertolini, P. Di Vecchia, M. Frau, A. Lerda, R. Marotta and I. Pesando, Fractional D-branes and their gauge duals, hep-th/0011077

19. O. Aharony, A note on holgraphic interpretation of string theory backgrounds with varying flux, hep-th/0101013.

20. E. Caceres and R. Hernandez, Glueball masses for the deformed conifold theory, Phys. Lett. B 504, 64 (2001) [hep-th/0011204].

21. J. P. Gauntlett, N. Kim and D. Waldram, M-fivebranes wrapped on supersymmetric cycles, hepth/0012195.

22. I.R. Klebanov and E. Witten, Superconformal field theory on threebranes at a Calabi-Yau singularity, Nucl. Phys. B536, 199 (1998) [hep-th/9807080].

23. S.S. Gubser and I.R. Klebanov, Baryons and domain walls in an $N=1$ superconformal gauge theory, Phys. Rev. D58, 125025 (1998), [hep-th/9808075].

24. I.R. Klebanov and N. Nekrasov, Gravity duals of fractional branes and logarithmic RG flow, Nucl. Phys. B574, 263 (2000), [hep-th/9911096].

25. I.R. Klebanov and A.A. Tseytlin, Gravity duals of supersymmetric $S U(N) \times S U(N+m)$ gauge theories, Nucl. Phys. B578, 123 (2000) [hep-th/0002159].

26. T. Eguchi and A.J. Hanson, Asymptotically flat self-dual solutions to Euclidean gravity, Phys. Lett. B74, 249 (1978).

27. K. Becker and M. Becker, M-theory on eight-manifolds, Nucl. Phys. B 477, 155 (1996) [hepth/9605053].

28. M.J. Duff, J.M. Evans, R.R. Khuri, J.X. Lu and R. Minasian, The octonionic membrane, Phys. Lett. B412, 281 (1997) [hep-th/9706124].

29. S.W. Hawking and M.M. Taylor-Robinson, Bulk charges in eleven dimensions, Phys. Rev. D58 025006 (1998) [hep-th/9711042].

30. K. Becker, A note on compactifications on Spin(7)-holonomy manifolds, hep-th/0011114.

31. C.P. Herzog and I.R. Klebanov, Gravity duals of fractional branes in various dimensions, hepth/0101020.

32. C. P. Herzog and P. Ouyang, Fractional D1-branes at finite temperature, hep-th/0104069.

33. A.S. Dancer and A. Swann, Hyperkähler metrics of cohomogeneity one, J. Geometry and Physics, 21, 218 (1997).

34. R.L. Bryant and S. Salamon, On the construction of some complete metrics with exceptional holonomy, Duke Math. J. 58, 829 (1989).

35. G.W. Gibbons, D.N. Page and C.N. Pope, Einstein metrics on $S^{3}, \mathbf{R}^{3}$ and $\mathbf{R}^{4}$ bundles, Commun. Math. Phys. 127, 529 (1990).

36. M. Cvetič, G.W. Gibbons, H. Lü and C.N. Pope, Supersymmetric M3-branes and $G_{2}$ manifolds, hep-th/0106031.

37. A. Brandhuber, J. Gomis, S.S. Gubser and S. Gukov, Gauge Theory at Large $N$ and New $G_{2}$ Holonomy Metrics, hep-th/0106034.

38. H. Kanno and Y. Yasui, On Spin(7) holonomy metric based on $S U(3) / U(1)$, hep-th/0108226.

39. M. Cvetič, G. W. Gibbons, H. Lü and C. N. Pope, Cohomogeneity one manifolds of Spin(7) and G(2) holonomy, hep-th/0108245.

40. S. Gukov and J. Sparks, M-theory on Spin(7) manifolds. I, hep-th/0109025. 
41. M.B. Stenzel, Ricci-flat metrics on the complexification of a compact rank one symmetric space, Manuscripta Mathematica, 80, 151 (1993).

42. P. Candelas and X. C. de la Ossa, Comments On Conifolds, Nucl. Phys. B 342, 246 (1990).

43. M. Atiyah and E. Witten, M-theory dynamics on a manifold of $G(2)$ holonomy, hep-th/0107177.

44. B. S. Acharya, On realising $N=1$ super Yang-Mills in $M$ theory, hep-th/0011089.

45. M. Atiyah, J. Maldacena and C. Vafa, An M-theory flop as a large $n$ duality, hep-th/0011256.

46. J. D. Edelstein and C. Nuñez, D6 branes and M-theory geometrical transitions from gauged supergravity, JHEP 0104, 028 (2001) [hep-th/0103167].

47. M. Aganagic and C. Vafa, Mirror symmetry and $a$ G(2) flop, hep-th/0105225.

48. M. Cvetič, H. Lü and C.N. Pope, Massless 3-branes in M-theory, hep-th/0105096. 